\title{
High-Resolution Transmission Electron Microscopy of Superconducting and Non-Superconducting Phases in a Bi-Sr-Cu-O System
}

\author{
By Yoshio Matsui*1, Shunji Takekawa*1, Kohji Kishio*2, Akihiro Umezono*3, \\ Sachiko Nakamura*4, Chusei Tsuruta*5 and Katsuhiko Ibe*6
}

\begin{abstract}
The superconducting phase (A) and three non-superconducting phases (B to $\mathbf{D}$ ) which exist in a $\mathrm{Bi}-\mathrm{Sr}-\mathrm{Cu}-\mathrm{O}$ system, with crystal structures closely related to each other, are examined by high-resolution transmission electron microscopy. A monoclinic modulated structure with periodical compression and expansion of $(\mathrm{BiO})_{2}$ layers in the $\mathrm{A}$ phase, $\mathrm{Bi}_{2.5} \mathrm{Sr}_{1.5} \mathrm{CuO}_{y}$, is confirmed. Cooperative atom displacements are induced to form terraced $(\mathrm{BiO})_{2}$ layers in the $\mathrm{B}$ phase, $\mathrm{Bi}_{17} \mathrm{Sr}_{16} \mathrm{Cu}_{7} \mathrm{O}_{y}$. The vertical shear planes are considered to contain few $\mathrm{Bi}$ atoms and are inserted regularly at every eighth lattice planes. Both $\mathrm{C}$ - and D-phases contain vertical slabs which also contain few Bi atoms. The C-phase, $\mathrm{Bi}_{4} \mathrm{Sr}_{8} \mathrm{Cu}_{5} \mathrm{O}_{y}$, has a commensurate superstructure with regular and straight arrangements of the slabs, while the D-phase, $\mathrm{Bi}_{2} \mathrm{Sr}_{3} \mathrm{Cu}_{2} \mathrm{O}_{y}$, has an incommensurate superstructure with wavy fluctuations of the slabs.
\end{abstract}

(Received February 10, 1990)

Keywords: superconducting oxides, modulated structures, high-resolution transmission electron microscopy, incommensurate superstructure, bismuth-strontium-copper-oxygen system

\section{Introduction}

Superconducting oxide containing bismuth (Bi) as one of its main components was first discovered in a $\mathrm{Bi}-\mathrm{Sr}-$ $\mathrm{Cu}-\mathrm{O}$ system by Michel et $a .^{(1)}$ and, independently, by Akimitsu et al. ${ }^{(2)}$ This compound showed superconductivity below $20 \mathrm{~K}$ and was later identified to be ideally $\mathrm{Bi}_{2} \mathrm{Sr}_{2} \mathrm{CuO}_{y}{ }^{(3)}$, although electron probe microanalysis (EP$\mathrm{MA})$ showed that the ratio of $\mathrm{Bi}, \mathrm{Sr}$ and $\mathrm{Cu}$ is close to $2.5: 1.5: 1$. The chemical composition of the compound is, therefore, described as $\mathrm{Bi}_{2+x} \mathrm{Sr}_{2-x} \mathrm{CuO}_{y}{ }^{(4)}$. Maeda et al. ${ }^{(5)}$ later obtained two high- $T_{\mathrm{c}}$ superconducting phases in a $\mathrm{Bi}-\mathrm{Sr}-\mathrm{Ca}-\mathrm{Cu}-\mathrm{O}$ system, which were later identified to be ideally $\mathrm{Bi}_{2} \mathrm{Sr}_{2} \mathrm{Ca}_{2} \mathrm{Cu}_{3} \mathrm{O}_{y}$ (high- $T_{\mathrm{c}}$ phase; $\left.T_{\mathrm{c}}=110 \mathrm{~K}\right)^{(6)}$ and $\mathrm{Bi}_{2} \mathrm{Sr}_{2} \mathrm{CaCu}_{2} \mathrm{O}_{y}$ (low- $T_{\mathrm{c}}$ phase; $\left.T_{\mathrm{c}}=80 \mathrm{~K}\right)^{(7)}$, respectively. These $\mathrm{Bi}$-based superconducting oxides are conveniently described simply by the ideal ratio of $\mathrm{Bi}, \mathrm{Sr}, \mathrm{Ca}$ and $\mathrm{Cu}$ as 2223,2212 and 2201 for the phases with $T_{\mathrm{c}}=110,80$ and $20 \mathrm{~K}$, respectively. They commonly have basic crystal structures consisting of alternate stacking of the $(\mathrm{BiO})_{2}$ layers and the perovskite-like slabs ${ }^{(4)(6)-(8)}$. Each perovskite-like slab contains one, two and three $\mathrm{Cu}$ -

*1 National Institute for Research in Inorganic Materials, 1-1 Namiki, Tsukuba-shi, Ibaraki 305, Japan.

*2 Department of Industrial Chemistry, The University of Tokyo, 7 3-1 Hongo, Bunkyo-ku, Tokyo 113, Japan.

*3 Fuji Laboratory, Tokai Carbon Ltd., Subashiri, Oyama-cho, Sunto-gun, Shizuoka 410-14, Japan.

*4 Hitachi Scientific Instrument Center, 26-27 Sakamachi, Shinjukuku, Tokyo 150, Japan.

*5 Hitachi Naka Works, 882 Ichige, Katsuta-shi, Ibaraki 312, Japan.

*6 EM Group, JEOL Ltd., Musashino, Akishima-shi, Tokyo 196, Japan. planes in the 2201, 2212 and 2223 phases. For instance, the 2201 phase has a tetragonal substructure with $a=b=0.54$ and $c=2.5 \mathrm{~nm}$ and has the layer sequences along the $c$ axis as follows:

$$
(\mathrm{BiO})_{2} \mathrm{SrO} \mathrm{CuO}_{2} \mathrm{SrO}(\mathrm{BiO})_{2} \mathrm{SrO} \mathrm{CuO}_{2} \mathrm{SrO}(\mathrm{BiO})_{2} \text {. }
$$

Both 2212 and 2223 phases also have tetragonal substructures with longer $c$ parameters of 3.06 and $3.6 \mathrm{~nm}$, respectively, due to the additional insertions of $\mathrm{CuO}_{2}$ and $\mathrm{Ca}$ layers in the perovskite-like slabs.

High-resolution transmission electron microscope (HRTEM) studies ${ }^{(9)-(13)}$ of the series of Bi-based superconductors, however, showed that they in fact have strongly modulated structures with large periodic distortions of lattice planes from the basic substructures mentioned above. Models of the modulated structure in the 2212 phase were soon proposed ${ }^{(14)(15)}$ based on the computer simulation of the HRTEM images. Similar modulated structures were found in both 2223 and 2201 phases $^{(11)(12)}$. Existences of twins and intergrowth defects are also detected by HRTEM experiments ${ }^{(13)}$.

Although $T_{\mathrm{c}}$ value of $\mathrm{Bi}_{2+x} \mathrm{Sr}_{2-x} \mathrm{CuO}_{y}$ phase is not so high, basic investigation of the $\mathrm{Bi}-\mathrm{Sr}-\mathrm{Cu}-\mathrm{O}$ system is considered to be quite important, because it is the simplest system producing Bi-based superconductors. Systematic study of the $\mathrm{Bi}-\mathrm{Sr}-\mathrm{Cu}-\mathrm{O}$ system was recently made by Takekawa et al. ${ }^{(4)}$ and the phase diagram as shown in Fig. 1 was proposed. There are totally four phases inside the triangular phase diagram. The A-phase stands for the superconducting 2201 phase mentioned above. The B-phase exists very close to the A-phase and contains slightly more $\mathrm{Sr}$ and less $\mathrm{Bi}$ atoms as compared to the A-phase. The remaining two phases (C and D), on the other hand, contain much more $\mathrm{Sr}$ and $\mathrm{Cu}$ as com- 


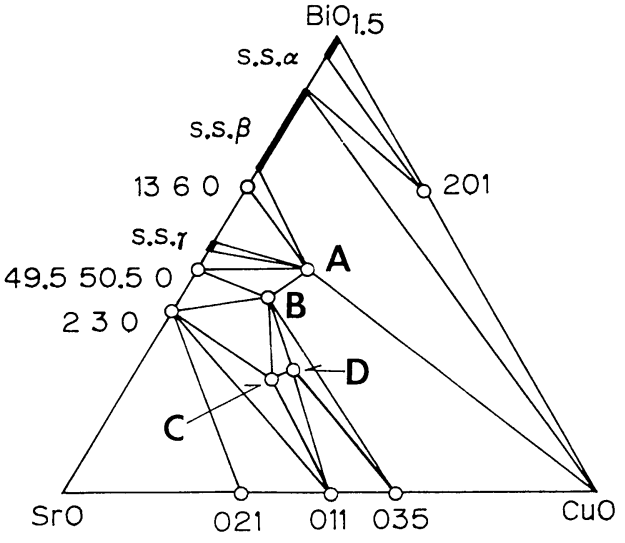

Fig. 1 Phase diagram of the $\mathrm{BiO}_{1.5}-\mathrm{SrO}-\mathrm{CuO}$ system after Takekawa et $a l .{ }^{(4)}$. There are four phases, A to D, in the system, where A stands for the superconducting 2201 phase. To keep consistency with a previous paper on the TEM observations ${ }^{(17)}, \mathrm{C}$ and D-phases are mutually interchanged from the original diagram by Takekawa et $a .^{(4)}$.

pared the A and B phases. Quite similar phase diagram including four different phases was also reported by Ikeda et al. ${ }^{(16)}$ Chemical compositions of these four phases are considered to be $\mathrm{Bi}_{2+x} \mathrm{Sr}_{2-x} \mathrm{CuO}_{y}(\mathrm{~A}), \mathrm{Bi}_{17} \mathrm{Sr}_{16} \mathrm{Cu}_{7} \mathrm{O}_{y}$ (B),
$\mathrm{Bi}_{4} \mathrm{Sr}_{8} \mathrm{Cu}_{5} \mathrm{O}_{y}$ (C) and $\mathrm{Bi}_{2} \mathrm{Sr}_{3} \mathrm{Cu}_{2} \mathrm{O}_{y}$ (D), respectively ${ }^{(4)(16)}$ Here, the assignments of the $\mathrm{C}$ - and $\mathrm{D}$-phases are interchanged to each other from the original diagram of Takekawa et $a l .{ }^{(4)}$ in order to keep consistency with our previous paper on the HRTEM observations ${ }^{(17)}$ of these four phases. According to the previous TEM observations $^{(17)}$, the $\mathrm{B}$ to $\mathrm{D}$ phases all exhibit characteristic electron diffraction patterns containing satellite reflections as shown in Fig. 2(b) to (d), suggesting the formation of some long-period superstructures closely related to the 2201 superconducting A-phase which exhibits electron diffraction pattern as shown in (a). Detailed structure analyses of these non-superconducting phases are, however, not yet presented. In the present study, we reexamined the structures of the four phases A to D in more detail by means of high-resolution transmission electron microscopy (HRTEM) to get more detailed structure models.

\section{Experimental}

Sintered blocks of the A- to D-phases are prepared by solid state reaction methods. Powders of $\mathrm{Bi}_{2} \mathrm{O}_{3}, \mathrm{SrCO}_{3}$ and $\mathrm{CuO}$ of $3 \mathrm{~N}$ grade were used as starting materials. They are mixed by appropriate mole ratio and prefired at
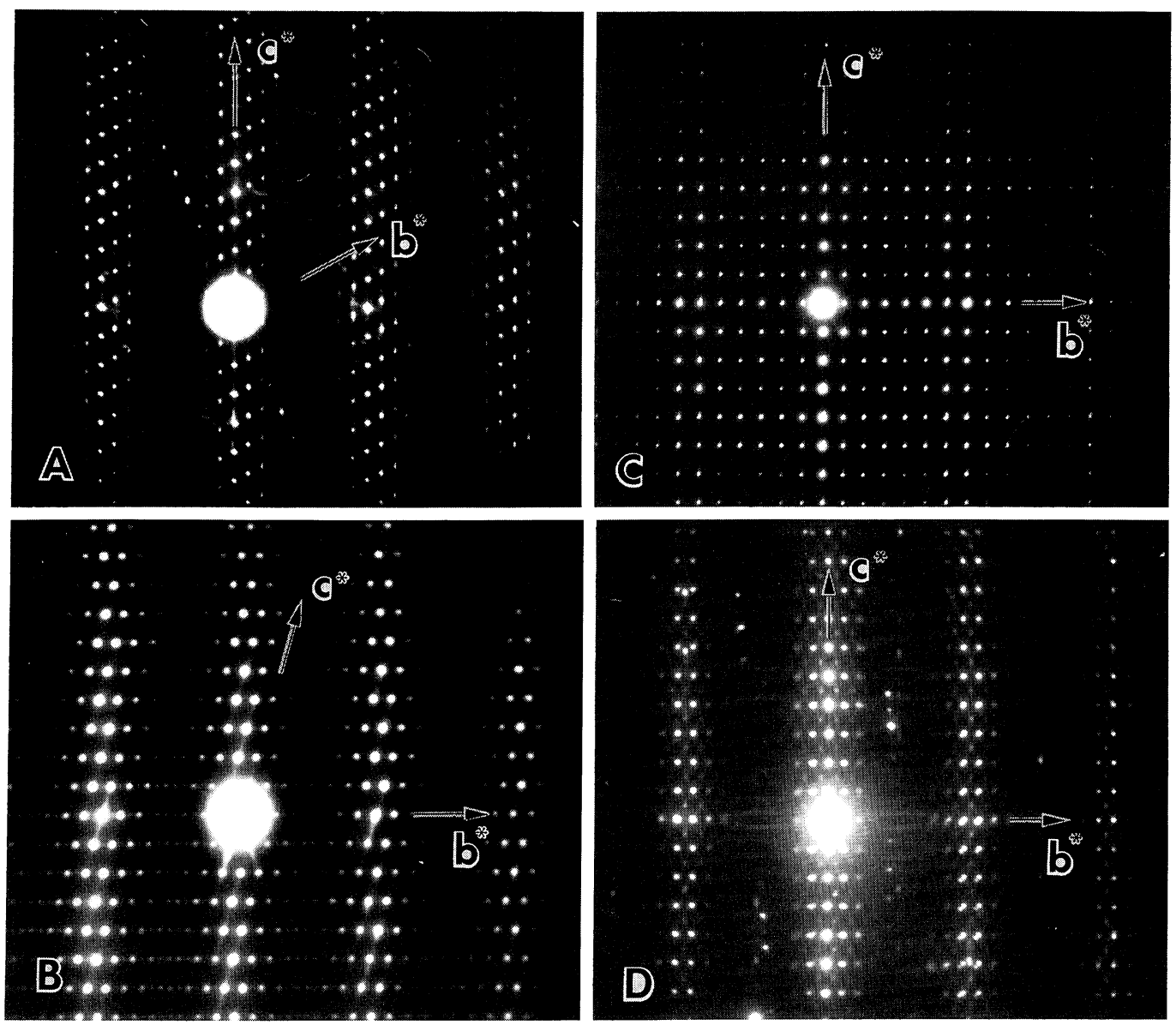

Fig. 2 The $b^{*}-c^{*}$ electron diffraction patterns ${ }^{(17)}$ of the four phases $\mathrm{A}$ to $\mathrm{D}$ in the phase diagram in Fig. 1 . The pattern in (a) corresponds to the superconducting A-phase, while the corresponding diffraction patterns from the other three non-superconducting phases, B to D, are shown in (b) to (d), respectively. 
700 to $760^{\circ} \mathrm{C}$ in air for about $12 \mathrm{~h}$. They are finally fired at 750 to $1050^{\circ} \mathrm{C}$ for 2 to 3 days and then rapidly cooled to room temperature. The obtained blocks were gently crushed in $\mathrm{CCl}_{4}$ and dispersed on holey carbon supporting films for HRTEM observations.

Single crystals of the B-phase are especially prepared by the floating-zone (FZ) method. Detailed procedures will be reported elsewhere ${ }^{(18)}$. The obtained rods of the Bphase are composed of the single crystalline plates elongating in the $a$ axis. They are molded in the epoxy resin and were cut directly by using a diamond knife equipped to the ultramicrotome (Reichert-Nissei; Ultracut-N).

HRTEM observations are made mainly by 1) JEOL JEM-4000EX type (400 kV) and 2) Hitachi H-9000 type $(300 \mathrm{kV})$ of high-resolution TEMs. Point-to-point resolution of these machines is expected to be around 0.17 to $0.19 \mathrm{~nm}$.

\section{Results}

\section{A-phase $\left(\mathrm{Bi}_{2+x} \mathrm{Sr}_{2-x} \mathrm{CuO}_{y}\right)$}

From the characteristic arrangements of satellite reflection spots in Fig. 2(a), the unit-cell of the superstructure of the superconducting A-phase is considered to be Bcentered monoclinic with $a_{\mathrm{m}}=a=0.54, b_{\mathrm{m}}=4.8 b=2.6$, $c_{\mathrm{m}}=c=2.8 \mathrm{~nm}$ and $\alpha=113$ degree. High-resolution transmission electron microscope image projected along the $a$ axis is shown in Fig. 3(a). The image contrasts are similar to those reported in the previous paper ${ }^{(12)}$. Double lines of dark contrast are considered to be $(\mathrm{BiO})_{2}$ layers. Periodic change of the dark contrast and also the fluctuation of $\mathrm{Bi}$ positions are observed in the $(\mathrm{BiO})_{2}$ layers. It is therefore considered that periodical compressions and expansions of $\mathrm{Bi}-\mathrm{Bi}$ distance is induced in the $(\mathrm{BiO})_{2}$ layers. This indicates that the $\mathrm{Bi}$-concentrated and $\mathrm{Bi}$-deficient bands similar to those observed in $\mathrm{Bi}_{2} \mathrm{Sr}_{2} \mathrm{CaCu}_{2} \mathrm{O}_{y}(2212)$ phase ${ }^{(9)(10)}$ are formed, as marked by " $\mathbf{B}$ " and " $\mathbf{D}$ " in Fig. 3(a). These Bi-concentrated and Bi-deficient bands are arranged to form a monoclinic superstructure, as expected from the corresponding electron diffraction pattern in Fig. 2(a). Figure 3(a) also shows that the centers of Bi-concentrated bands of the upper and the lower (BiO) plane are slightly displaced to each other along the $b$ direction. Onoda and Sato ${ }^{(19)}$ found the crystal with commensurate periodicity of $b_{\mathrm{m}}=5 b$ and proposed a similar monoclinic modulated structure based on the Xray structure analysis. Schematic representation of the structure model after Onoda and Sato ${ }^{(19)}$ is shown in Fig. 3(b). Although no atom replacements of Bi positions are assumed in the structure model of Fig. 3(b), we consider that some of Bi atoms in the Bi-deficient bands, $\mathbf{D}$, are replaced by the lighter ones such as $\mathrm{Sr}$ or $\mathrm{Cu}$. Detailed analysis of image contrast by means of computer simulations is in progress. HRTEM image in Fig. 3(a) also shows that a slight local fluctuation of the direction of the $c$ axes are induced and this indicates that $\alpha$ parameter of the monoclinic superlattice can fluctuate locally.
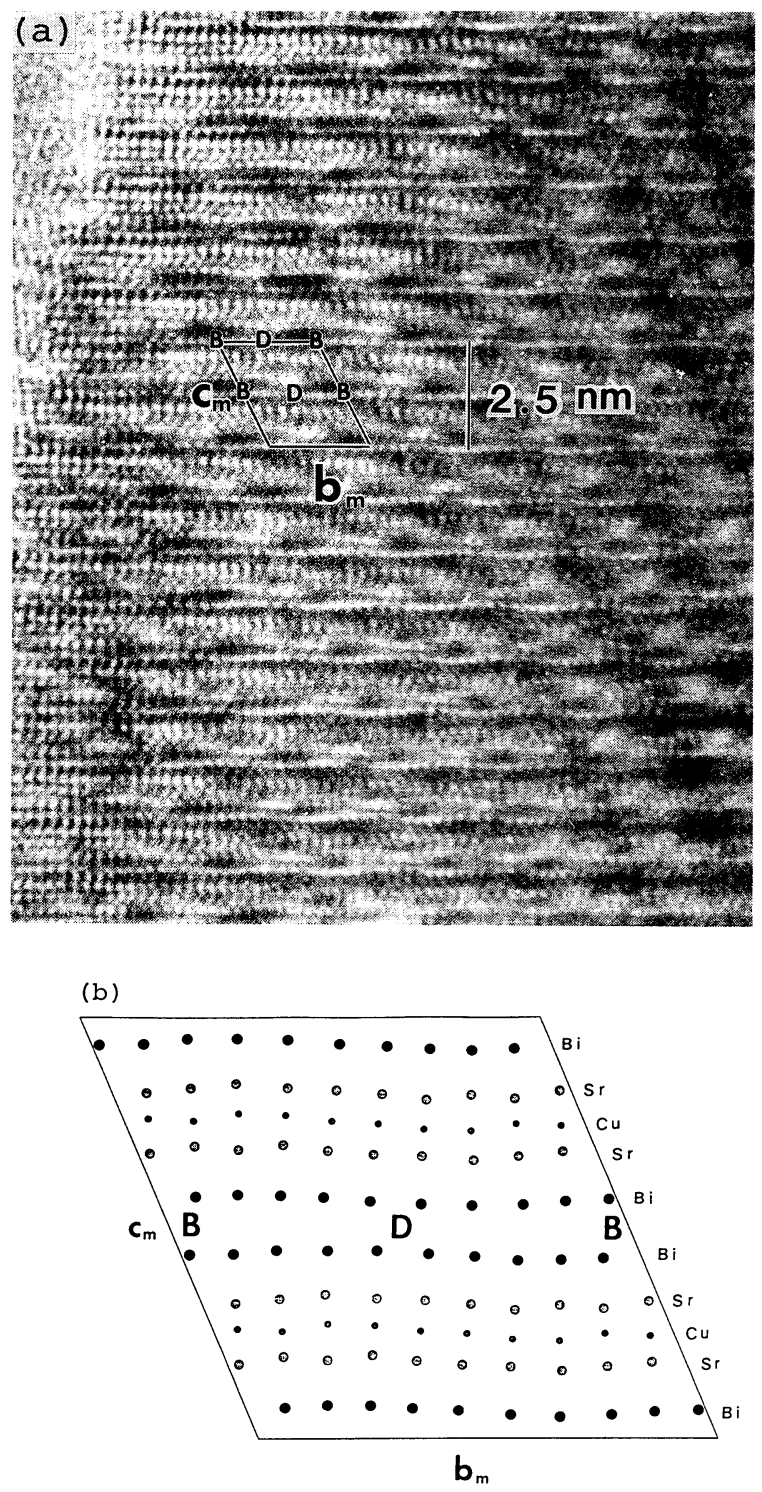

Fig. 3 (a) HRTEM image of the superconducting A-phase showing the monoclinic modulation structure. The symbols "B' and "D'" stand for the $\mathrm{Bi}$-concentrated and $\mathrm{Bi}$-deficient bands, respectively, in the $(\mathrm{BiO})_{2}$ layers. The model of monoclinic modulation structure approximated by five-times commensurate structure ${ }^{(19)}$ is shown in (b).

\section{B-phase $\left(\mathrm{Bi}_{17} \mathrm{Sr}_{16} \mathrm{Cu}_{7} \mathrm{O}_{y}\right)$}

The $b^{*}-c^{*}$ electron diffraction pattern from the Bphase obtained by the solid state reactions $s^{(17)}$ is shown in Fig. 2(b) and it clearly indicates that the $c^{*}$ is not normal to the $b^{*}$ direction. This means that the superstructure has monoclinic symmetry. The unit cell parameters read out from the diffraction pattern are $a_{\mathrm{s}}=0.54, b_{\mathrm{s}}=2.0$ $c_{\mathrm{s}}=2.5 \mathrm{~nm}$ and $\alpha=104^{\circ}$. Arrangements of satellite spots along $b^{*}$ suggest that the B-phase has an incommensurate periodicity in the $b$ direction. The corresponding lattice image reported previously suggested that each $(\mathrm{BiO})_{2}$ layer is not a complete continuous sheet but suffers from periodic cooperative displacements along the $c$ axis to form stepped (or terraced) $(\mathrm{BiO})_{2}$ layers $^{(17)}$. Quality of the lattice image was, however, not good 

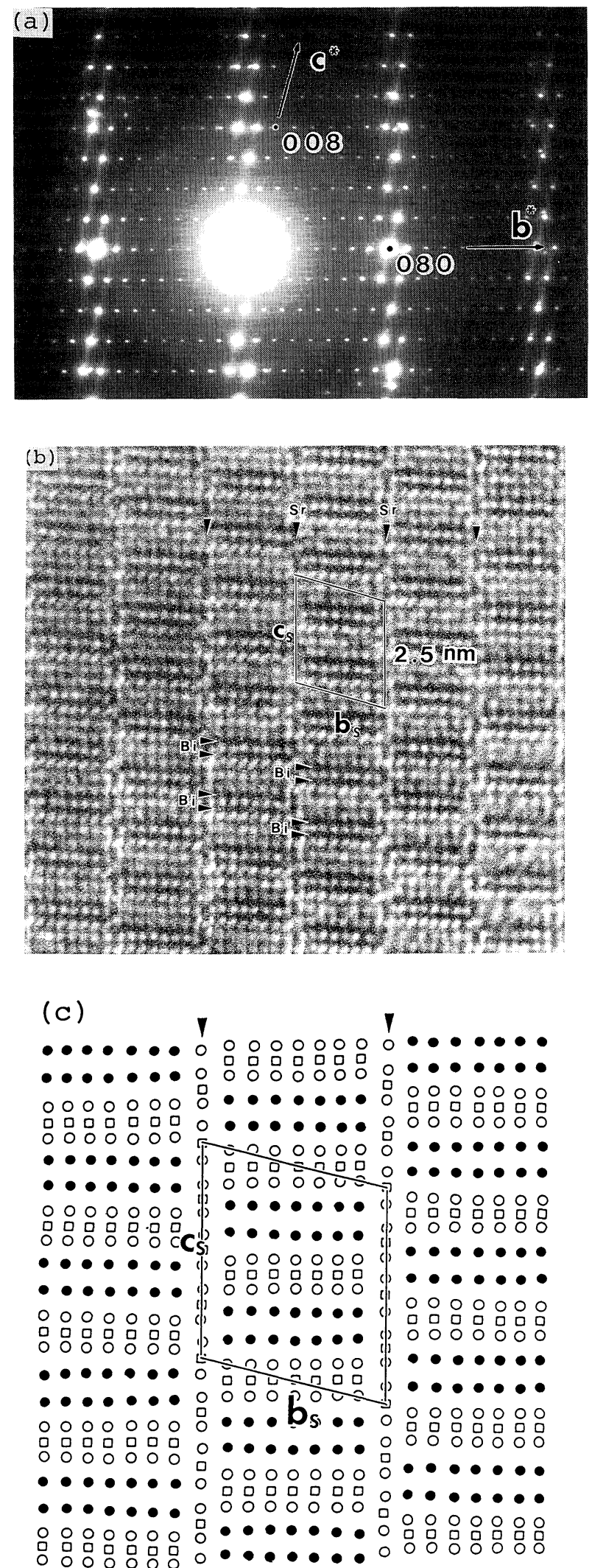

Fig. 4 The $b^{*}-c^{*}$ electron diffraction pattern from the single crystal of the B-phase is shown in (a). HRTEM image of the B-phase projected along the a direction is shown in (a). The corresponding HRTEM image is shown in (b) and the formations of terraced $(\mathrm{BiO})_{2}$ layers are clearly observed. The vertical shear planes indicated by arrowheads are considered to consist of $\mathrm{Sr}$ and $\mathrm{Cu}$ atoms. Schematic model of the structure is shown in (c). Solid circles, open circles and open squares stand for $\mathrm{Bi}, \mathrm{Sr}$ and $\mathrm{Cu}$ positions, respectively. enough to obtain detailed structure model of the Bphase. One serious problem to obtain the HRTEM image from the B-phase is that the chance to meet the crystal with [100] orientation is very low because of the strong cleavage of the crystal. In the present study, therefore, we used a single crystalline sample of the B-phase prepared by the floating-zone (FZ) method $^{(18)}$ and tried to prepare the thin foil for TEM observation by use of ultramicrotome equipped with a diamond knife. The chance to meat the crystals of [100] orientation increases greatly by this method. The $b^{*}-c^{*}$ electron diffraction pattern from the single crystalline sample is shown in Fig. 4(a). The satellite reflection spots along the $b^{*}$ direction are almost equally spaced and there are seven satellite spots between the central and the 020 reflection from the basic structure. This indicates that cooperative atom displacements which cause stepped $(\mathrm{BiO})_{2}$ layers, are induced quite regularly with a periodicity of $4 b=2.1$ $\mathrm{nm}$ in the single crystalline sample, while some irregularity of atom displacements causes an incommensurate periodicity of about $3.8 b=2.0 \mathrm{~nm}$ in the polycrystalline sample of the B-phase. The lattice parameters expected from the electron diffraction pattern in Fig. 4(a) are $a_{\mathrm{s}}=0.54, b_{\mathrm{s}}=2.2, c_{\mathrm{s}}=2.8$ and $\alpha=104^{\circ}$. The corresponding HRTEM image is shown in Fig. 4(b). The terraced $(\mathrm{BiO})_{2}$ double layers are clearly observed. The image also shows that there are vertical shear planes with contrasts weaker than the $(\mathrm{BiO})_{2}$ layers, as indicated by arrowheads. We consider that these shear planes consist mainly of $\mathrm{Sr}$ and $\mathrm{Cu}$ atoms. It is read out from the image that each terrace consists of seven lattice planes of a standard 2201 structure and the vertical shear planes of $\mathrm{Sr}$ and $\mathrm{Cu}$ are inserted at every eight lattice planes as shown in Fig. 4(c). In this model, we assume that each shear plane has a sequence of cations along the $c$ direction as,

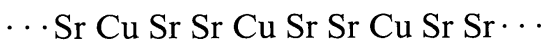

We consider that the model is fairly reasonable because the B-phase contains slightly less $\mathrm{Bi}$ as compared to the A-phase according to the phase diagram of Fig. 1. The ideal composition derived from the model is $\mathrm{Bi}_{14+x} \mathrm{Sr}_{17-x} \mathrm{Cu}_{9} \mathrm{O}_{y}$, which is close to the experimental composition $\mathrm{Bi}_{17} \mathrm{Sr}_{16} \mathrm{Cu}_{7} \mathrm{O}_{y}{ }^{(16)}$. Detailed analysis of the structure by means of single crystal $\mathrm{X}$-ray methods are in progress.

Formations of intergrowth defect due to the local changes of the periodicity of shear planes are observed as shown by the symbol ' $F$ ', in Fig. 5. Width of the shorter terraces is about $1.8 \mathrm{~nm}$, which is about $0.26 \mathrm{~nm}$ less than the width of the major terraces. We consider that one of the seven 2201 lattice plane is missed at these narrow terraces. Incommensurate periodicity observed in the previous polycrystalline sample of the B-phase ${ }^{(17)}$, obtained by the solid state reaction method, is probably due to the higher densities of such intergrowth defects in the crystals.

It is considered worth mentioning that we accidentally find the region where a micro-crack is introduced in the crystal of B-phase, as shown by an arrow in Fig. 6. Prob- 


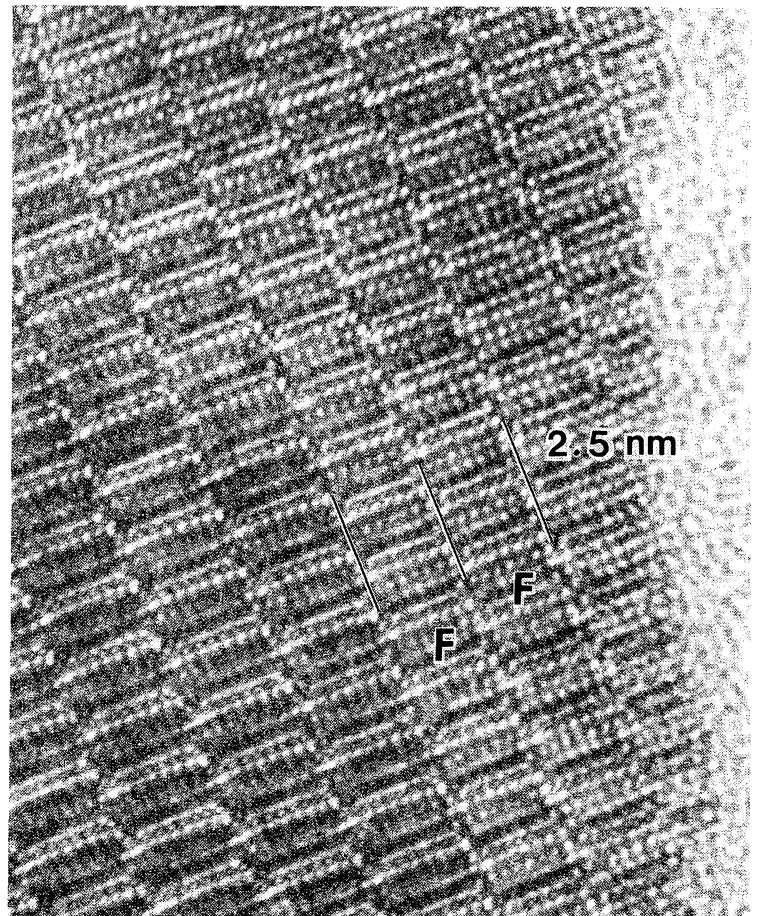

Fig. 5 HRTEM image of the intergrowth of narrow terraces, indicated by the symbol " $F$ ", in the B-phase, which is due to the shorter distance of the vertical shear planes of $\mathrm{Sr}$ and $\mathrm{Cu}$ where the cooperative displacements are induced.

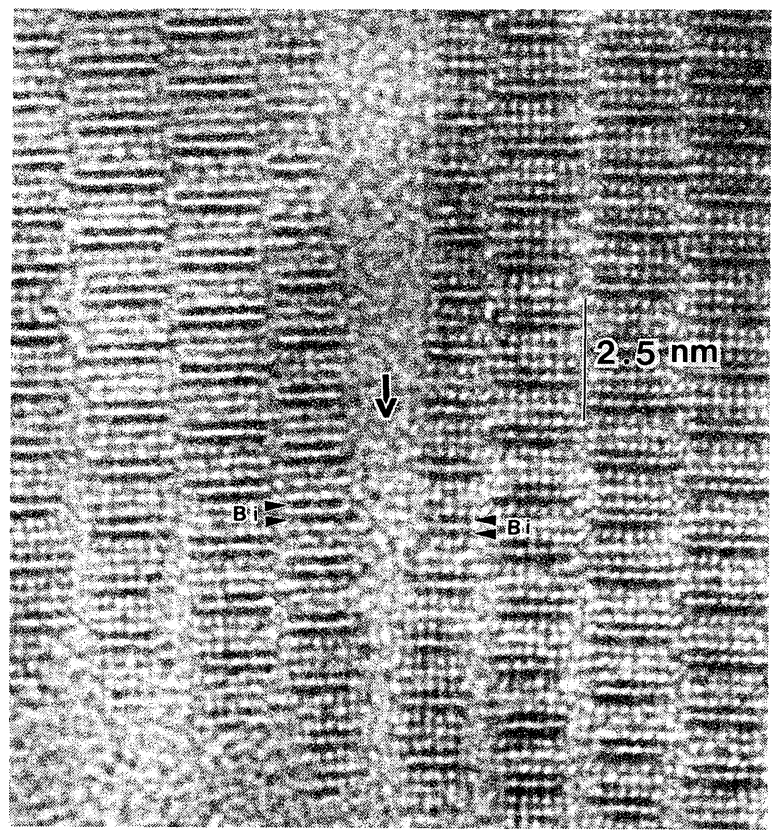

Fig. 6 HRTEM image of the B-phase showing the cracks which are introduced along the vertical sheet as indicated by an arrow. This indicates that the chemical bonding at the vertical sheet is much weaker than the bonding across the $(\mathrm{BiO})_{2}$ layers.

ably, the crack was formed during the sample was cut by the diamond knife. It is considered interesting that the crack is introduced along the shear plane parallel to the $c$ axis and not along the $(\mathrm{BiO})_{2}$ layers. In most of the $\mathrm{Bi}$ based superconductors and related materials, chemical bonding between the two $(\mathrm{BiO})$ planes is very weak and, therefore, the crystals usually have strong cleavage parallel to the $c$ plane. Image of the crack in Fig. 6, however, suggests that the vertical shear planes which are nearly normal to the $(\mathrm{BiO})_{2}$ layers tend to cleave and therefore the cleavage plane is normal to the $c$ plane in the B-phase.

\section{C-phase $\left(\mathrm{Bi}_{4} \mathrm{Sr}_{8} \mathrm{Cu}_{5} \mathrm{O}_{y}\right)$}

Both C- and D-phases exist very close to each other in the phase diagram in Fig. $1^{(4)(16)}$. The crystal structures of the C- and D-phases are, therefore, considered to be closely related to each other. Previous TEM observations $^{(17)}$ showed that $\mathrm{C}$ - and D-phases have commensurate and incommensurate superstructures, respectively, both derived from the basic structure of the superconducting A-phase.

The $b^{*}-c^{*}$ electron diffraction pattern of the C-phase shown in Fig. 2(c) exhibits quite regular arrangements of reflection spots indicating formation of commensurate superstructure $^{(17)}$. It has a face-centered orthorhombic structure with $a=0.54, b=3.4$ and $c=2.5 \mathrm{~nm}$. The corresponding HRTEM image is shown in Fig. 7(a). Double horizontal lines of dark contrast are considered to be $(\mathrm{BiO})_{2}$ like layers. The image clearly shows that the vertical slabs are inserted with a spacing of $b / 2=1.7 \mathrm{~nm}$. From the phase diagram in Fig. 1, it is expected that the $\mathrm{C}$-phase contains more $\mathrm{Sr}$ and $\mathrm{Cu}$ atoms as compared to the 2201 A-phase. We therefore consider that the vertical slabs are composed mainly of $\mathrm{Sr}$ and $\mathrm{Cu}$ atoms. Recently, Fuertes et al. ${ }^{(20)}$ reported results of X-ray crystal structure analysis of the compound with a composition $\mathrm{Bi}_{4} \mathrm{Sr}_{8} \mathrm{Cu}_{5} \mathrm{O}_{y}$, which possibly corresponds to the C-phase. According to the model proposed by them, there are vertical slabs which are composed of two vertical lines of $\mathrm{Sr}$ and single line of $\mathrm{Cu}$. Between the slabs, there are four lattice planes having structure of the 2201 phase, as schematically described in Fig. 7(b). The vertical lines of dark contrast indicated by arrowheads in Fig. 7(a) are considered to be the Sr planes of the vertical slabs. Detailed comparisons of this model and the HRTEM images by means of computer simulations are in progress.

\section{D-phase $\left(\mathrm{Bi}_{2} \mathrm{Sr}_{3} \mathrm{Cu}_{2} \mathrm{O}_{y}\right)$}

The D-phase, which contains slightly less $\mathrm{Sr}$ and $\mathrm{Cu}$ atoms as compared to the C-phase, exhibits the electron diffraction pattern shown in Fig. 2(d) with characteristic satellite reflection spots which corresponds to an incommensurate periodicity of about $2.3 \mathrm{~nm}$ in the $b$ direction. The corresponding HRTEM image is shown in Fig. 8(a) and it clearly shows that the vertical slabs similar to those observed in the C-phase are inserted in the structure. The vertical slabs are however not completely straight along the $c$ axis but tend to shift along the $b$ axis when they cross the $(\mathrm{BiO})_{2}$ layers. The vertical slabs, therefore, suffer from wavy arrangements and the lateral distance between the two slabs changes locally. The average distance of two vertical slabs, expected from the electron diffraction pattern, is about $2.3 \mathrm{~nm}$, which is longer than the corresponding distance, $1.7 \mathrm{~nm}$, in the C-phase. Such 


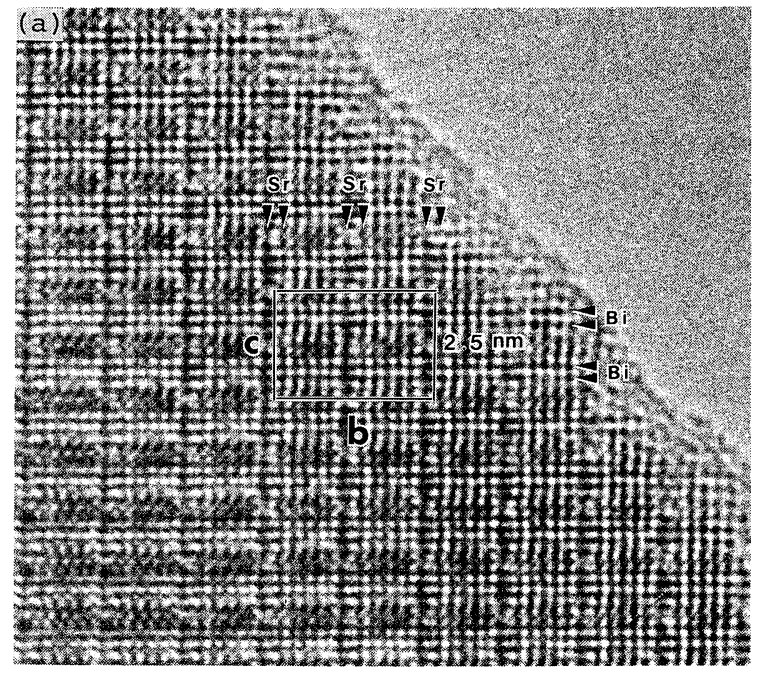

(b)

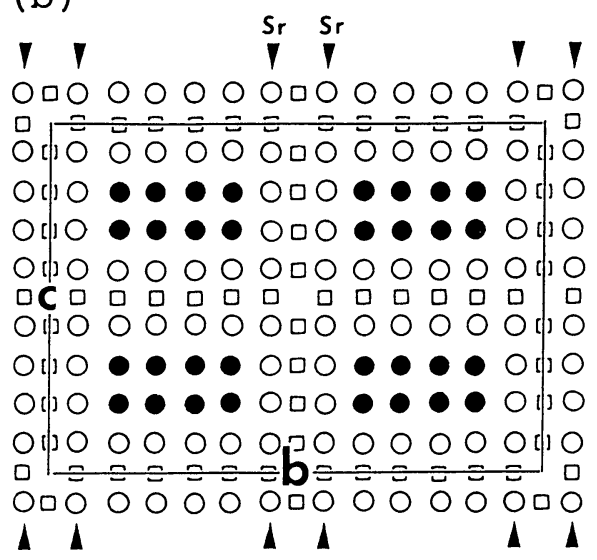

Fig. 7. HRTEM image of the commensurate structure of the C-phase is shown in (a). The vertical slabs, containing two $\mathrm{Sr}$ - and one $\mathrm{Cu}-$ planes, are inserted quite regularly with $1.7 \mathrm{~nm}$ spacing as indicated by arrowheads. Schematic diagram of the superstructure, drawn after Fuertes et al. ${ }^{(20)}$, is shown in (b). Solid circles, open circles and open squares stand for $\mathrm{Bi}, \mathrm{Sr}$ and $\mathrm{Cu}$ positions respectively.

wider distance of the slabs of $\mathrm{Sr}$ and $\mathrm{Cu}$ is consistent with the less $\mathrm{Sr}$ and $\mathrm{Cu}$ contents of the phase $\mathrm{D}$ as compared to the C-phase. We assume that the vertical slabs have local composition and structure similar to those of the Cphase. From the average distance of $2.3 \mathrm{~nm}$ mentioned above, we consider that, in average, there are seven or eight lattice planes of the 2201 structure between the two vertical slabs. If we assume the case of eight lattice planes of the 2201 structure, then it is expected that the D-phase has an ideal composition $\mathrm{Bi}_{16} \mathrm{Sr}_{24} \mathrm{Cu}_{14} \mathrm{O}_{y}$, which is close to the experimental composition $\mathrm{Bi}_{2} \mathrm{Sr}_{3} \mathrm{Cu}_{2} \mathrm{O}_{y}$. If we assume the case of seven 2201 lattice planes, we expect the ideal composition $\mathrm{Bi}_{14} \mathrm{Sr}_{22} \mathrm{Cu}_{13} \mathrm{O}_{y}$, which is also close to the experimental composition. The narrowest and the widest distances of the slabs observed in the present HRTEM image are about 2 and $3 \mathrm{~nm}$, which corespond to the ideal compositions $\mathrm{Bi}_{12} \mathrm{Sr}_{20} \mathrm{Cu}_{12} \mathrm{O}_{y}$ and $\mathrm{Bi}_{20} \mathrm{Sr}_{28} \mathrm{Cu}_{16} \mathrm{O}_{y}$, respectively. In this way, the structure of the D-phase is derived by the insertions of the slabs of $\mathrm{Sr}$ and $\mathrm{Cu}$, which suffer from wavy fluctuations to cause various widths of two

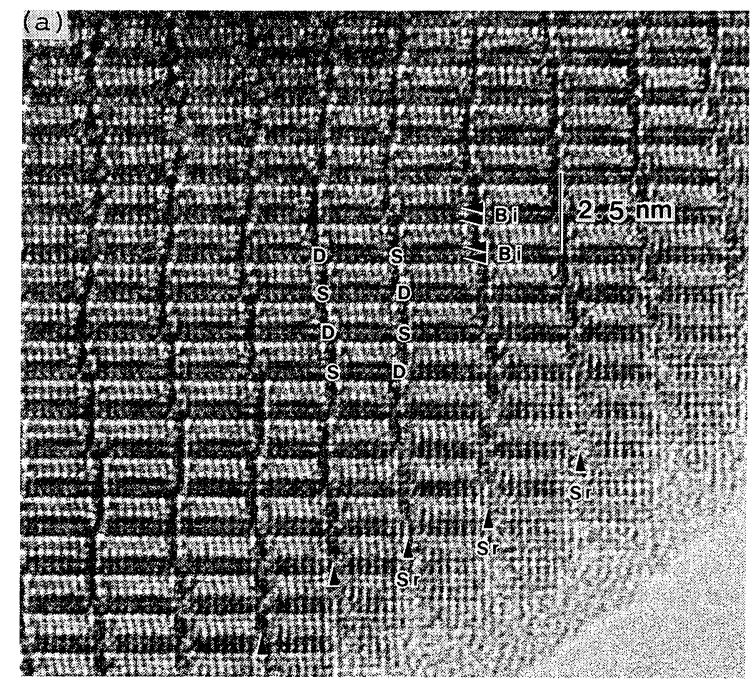

(b)

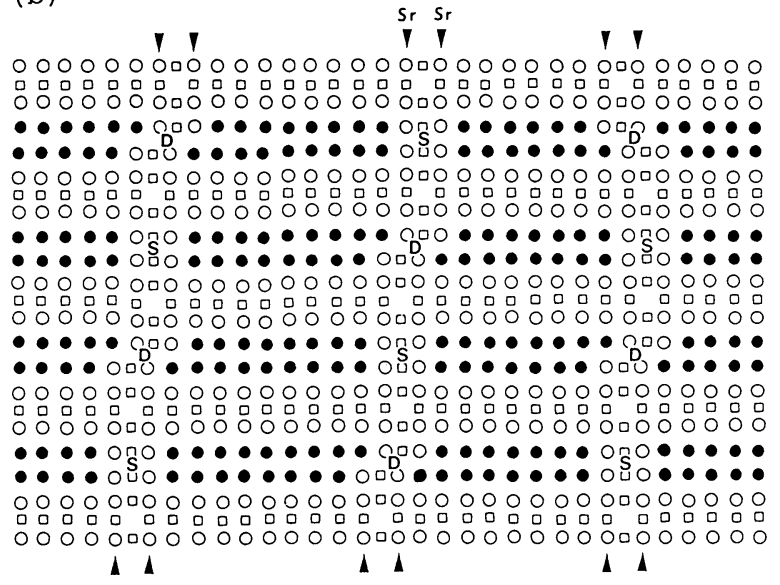

Fig. 8 HRTEM image of the incommensurate superstructure of the D-phase, showing irregular and wavy arrangements of the vertical slabs of $\mathrm{Sr}$ and $\mathrm{Cu}$, is shown in (a). The slabs suffer from lateral displacements at the regions marked by ' $D$ ', while no displacements are induced at "S'. Average spacing of the vertical planes are about $2.3 \mathrm{~nm}$, which is longer than the corresponding spacing $1.7 \mathrm{~nm}$ of the C-phase. Schematic diagram of the structure is shown in (b). Solid circles, open circles and open squares stand for $\mathrm{Bi}, \mathrm{Sr}$ and $\mathrm{Cu}$ positions, respectively.

slabs ranging from 2 to $3 \mathrm{~nm}$. A broad peak at about $d=2.5 \mathrm{~nm}$ observed in the powder X-ray diffraction chart $^{(4)}$ is considered to be due to such variations of the distance of the two vertical slabs in the D-phase. From the HRTEM image in Fig. 8(a), we can deduce some rules on the way of displacements of the vertical slabs. First, the probability of causing displacements, when the vertical bands and the $(\mathrm{BiO})_{2}$ layers are crossed, is almost 0.5. In Fig. 8(a), regions where no displacements are induced are marked by ' $\mathbf{S}$ ', while the regions of displacements are by "D'. It is clearly observed that " $\mathbf{S}$ " and " $D$ ', repeats alternately in both $b$ and $c$ directions. Second, the amount of displacement is always about $0.26 \mathrm{~nm}$, which corresponds to the 020 lattice distance of the substructure. Schematic diagram of the structure of the D-phase obtained by considering these 
rules of horizontal displacements of $(\mathrm{BiO})_{2}$ layers are given in Fig. 8(b). In this diagram, we assume that the displacements are induced in between the two (BiO) planes. More detailed analysis of the images are still in progress.

\section{Conclusions}

(1) Modulated structure of the superconducting A-phase, $\mathrm{Bi}_{2+x} \mathrm{Sr}_{2-x} \mathrm{CuO}_{y}$, is reexamined and the monoclinic superstructure, with periodic compression and expansion of the $(\mathrm{BiO})_{2}$ layers, is confirmed as shown in Fig. 3(a). Local fluctuations of the $\alpha$ parameter is observed.

(2) We also reexamined the structure of the B-phase, $\mathrm{Bi}_{17} \mathrm{Sr}_{16} \mathrm{O}_{7} \mathrm{O}_{y}$, in detail, using single crystal samples prepared by the floating-zone (FZ) method. HRTEM image in Fig. 4(b) clearly showed that each $(\mathrm{BiO})_{2}$ layer has a terraced structure, due to periodic cooperative displacements of $(\mathrm{BiO})_{2}$ layers along the $c$ direction. The vertical shear planes, where such displacements are induced, are inserted at every eight lattice plane. The crystal of the B-phase prepared by the FZ method has quite commensurate structure, although the B-phase obtained by the solid state reaction methods had an incommensurate structure. Intergrowth defects and crack in the B-phase are also observed.

(3) Both C- and D-phases have the superstructures derived by the formations of vertical slabs of $\mathrm{Sr}$ and $\mathrm{Cu}$ in the structure. The slabs are inserted quite regularly, with a spacing of $1.7 \mathrm{~nm}$, in the C-phase to form a commensurate superstructure. In the D-phase, on the other hand, the slabs are not straight along the $c$ direction but suffer from the displacements along the $b$ direction when they cross the $(\mathrm{BiO})_{2}$ layers. The lateral distance of two vertical bands vary between 2 and $3 \mathrm{~nm}$ to form an incommensurate superstructure. Some rules on the displacements of $(\mathrm{BiO})_{2}$ layers are discussed.

\section{Acknowledgments}

The authors would like to thank Prof. K. Kitazawa of Univ. of Tokyo, Dr. F. Nagata of Hitachi Ltd., Dr. S.
Horiuchi, Dr. S. Kimura, Dr. A. Yamamoto, Dr. F. Izumi of NIRIM for valuable discussions. They also thank Mr. Y. Kitami and Mr. M. Yokoyama for the support in HRTEM experiments.

\section{REFERENCES}

(1) C. Michel, M. Hervieu, M. M. Borel, A. Grandin, F. Deslandes, J. Provost and B. Rabeau: Z. Phys., B68 (1987), 421.

(2) J. Akimitsu, A. Yamazaki, H. Sawa and H. Fujiki: Jpn. J. Appl. Phys., 26 (1987), L2080.

(3) H. Fujiki, M. Sano, K. Tomimoto, H. Sawa, J. Akimitsu and N. Kitamura: Jpn. J. Appl. Phys., 27 (1988), L1044.

(4) S. Takekawa, A. Umezono and H. Nozaki: in preparation.

(5) H. Maeda, Y. Tanaka, M. Fukutomi and T. Asano: Jpn. J. Appl. Phys., 27 (1988), L209.

(6) H. W. Zandbergen, Y. K. Huang, M. J. V. Menken, J. N. Li, K. Kadowaki, A. A. Menovsky, G. Van Tendeloo and S. Amelinckx: Nature, 332 (1988), 620.

(7) E. Takayama-Muromachi, Y. Uchida, A. Ono, F. Izumi, M. Onoda, Y. Matsui, K. Kosuda, S. Takekawa and K. Kato: Jpn. J. Appl. Phys., 27 (1988), L365.

(8) J. M. Tarascon, Y. Le Page, P. Barboux, B. G. Bagley, L. H. Greene, W. R. McKinnon, G. W. Hull, M. Giroud and D. M. Huang: Phys. Rev., B37 (1988), 9382.

(9) Y. Matsui, H. Maeda, Y. Tanaka and S. Horiuchi: Jpn. J. Appl. Phys., 27 (1988), L361.

(10) Y. Matsui, H. Maeda, Y. Tanaka and S. Horiuchi: Jpn. J. Appl. Phys., 27 (1988), L372.

(11) Y. Matsui, S. Takekawa, H. Nozaki, A. Umezono, E. TakayamaMuromachi and S. Horiuchi: Jpn. J. Appl. Phys., 27 (1988), L1241.

(12) Y. Matsui, S. Takekawa, S. Horiuchi and A. Umezono: Jpn. J. Appl. Phys., 27 (1988), L1873.

(13) Y. Matsui, H. Maeda, Y. Tanaka, E. Takayama-Muromachi, S. Takekawa and S. Horiuchi: Jpn. J. Appl. Phys., 27 (1988), L827.

(14) S. Horiuchi, H. Maeda, Y. Tanaka and Y. Matsui: Jpn. J. Appl. Phys., 27 (1988), L1172.

(15) Y. Hirotsu, O. Tomioka, T. Ohkubo, N. Yamamoto, Y. Nakamura, S. Nagakura, T. Komatsu and K. Matsushita: Jpn. J. Appl. Phys., 27 (1988), L1869.

(16) Y. Ikeda, H. Ito, S. Shimomura, Y. Oue, K. Inaba, Z. Hiroi and M. Takano: Physica, C159 (1989), 93.

(17) Y. Matsui, S. Takekawa, H. Nozaki and A. Umezono: Jpn. J. Appl. Phys., 28 (1989), L602.

(18) K. Kishio et al.: private communications.

(19) M. Onoda and M. Sato: Solid State Commun., 67 (1988), 799.

(20) A. Fuertes, C. Miravitlles, J. Gonzalez-Calbet, M. Vallet-Regi, X. Obradors and J. Rodriguez-Carvajal: Physica, C157 (1989), 525. 